\begin{tabular}{|c|l|}
\hline Title & Residual stress distribution in rabbit limb bones \\
\hline Author(s) & Y amada, Satoshi; Tadano, Shigeru; Fujisaki, Kazuhiro \\
\hline Citation & $\begin{array}{l}\text { Journal of Biomechanics, 44(7), 1285-1290 } \\
\text { https://doi.org/10.1016/.jbiomech.2011.01.038 }\end{array}$ \\
\hline Issue Date & 2011-04 \\
\hline Doc URL & http://hdl.handle.net/2115/45598 \\
\hline Type & article(author version) \\
\hline File Information & JB44_1285-1290.pdf \\
\hline
\end{tabular}

Instructions for use 


\title{
Residual Stress Distribution in Rabbit Limb Bones
}

\author{
Satoshi YAMADA ${ }^{\mathrm{a}}$, Shigeru TADANO ${ }^{\mathrm{b},{ }^{*}}$, Kazuhiro FUJISAKI ${ }^{\mathrm{b}}$
}

${ }^{a}$ Division of Human Mechanical Systems and Design, Graduate School of Engineering, Hokkaido University, N13 W8, Kita-ku, Sapporo, Hokkaido 060-8628, Japan

$\mathrm{b}$ Division of Human Mechanical Systems and Design, Faculty of Engineering, Hokkaido University, N13 W8, Kita-ku, Sapporo, Hokkaido 060-8628, Japan

*Corresponding Author:

Shigeru TADANO, PhD

Professor, Division of Human Mechanical Systems and Design, Faculty of Engineering, Hokkaido University

N13 W8, Kita-ku, Sapporo, Hokkaido 060-8628, Japan

Tel/Fax: +81-11-7066405

E-mail: tadano@eng.hokudai.ac.jp

Word count: 2908 words (Introduction through Acknowledgement)

Manuscript Type: Original Article

Keywords: Residual Stress, Cortical Bone, Rabbit Limb Bones, Osteon, X-ray Diffraction 


\section{Abstract}

The presence of the residual stresses in bone tissue has been noted and the authors have reported that there are residual stresses in bone tissue. The aim of our study is to measure the residual stress distribution in the cortical bone of the extremities of vertebrates and to describe the relationships with the osteon population density. The study used the rabbit limb bones (femur, tibia/fibula, humerus, and radius/ulna) and measured the residual stresses in the bone axial direction at anterior and posterior positions on the cortical surface. The osteons at the sections of the measurement positions were observed by microscopy. As a result, the average stresses at the hindlimb bones and the forelimb bones were $210 \mathrm{MPa}$ and $149 \mathrm{MPa}$, respectively. In the femur, humerus, and radius/ulna, the residual stresses at the anterior position were larger than those at the posterior position, while in the tibia, the stress at the posterior position was larger than that at the anterior position. Further, in the femur and humerus, the osteon population densities in the anterior positions were larger than those in the posterior positions. In the tibia, the osteon population density in the posterior position was larger than that in the anterior position. Therefore, tensile residual stresses were observed at every measurement position in the rabbit limb bones and the value of residual stress correlated with the osteon population density $(r=0.55, P<0.01)$. (234 words) 


\section{Introduction}

Living tissue, like blood vessels, is subject to residual stresses (Fung, 1990).

The presence of residual stresses in bone has also been noted (Tadano and Okoshi, 2006). A $\sin ^{2} \psi$ method of $\mathrm{X}$-ray diffraction as the measurement method of the residual stress in bone tissue has been proposed and residual stresses in the bone tissue of the bovine femurs have been reported (Yamada and Tadano, 2010). The residual stress is defined as the stress that remains in bone tissue without any external forces. This previous study of bovine femoral diaphyses showed that the residual stresses in the bone axial direction were tensile, and the residual stresses in the bone tissue were discussed as a factor in the tissue strength.

Cortical bone has a hierarchal and composite structure formed by hydroxyapatite (HAp) like mineral particles and collagen matrix. The HAp in bone tissue has a hexagonal crystalline structure, and X-ray diffraction can be used to measure the interplanar spacings of HAp crystals (Fujisaki et al., 2006; Gupta et al., 2006; Almer and Stock, 2007; Fujisaki and Tadano, 2007; Tadano et al., 2008; Giri et al., 2009). When bone tissue deforms, the displacement of the lattice planes of the HAp crystals change almost proportionally. It has been shown that the distance between the lattice planes of the HAp crystals change proportionally to the deformation of the bone 
tissue (Fujisaki and Tadano, 2007). The HAp crystal strain can be calculated by the deformation of the interplanar spacing compared with a reference state (Fujisaki et al., 2006; Tadano et al., 2008). Based on this, the residual stresses in bone tissue can be measured using the $\sin ^{2} \psi$ method of X-ray diffraction.

In general, the residual stress is produced in a material by the indeterminate structure. It is well known that bone is usually replaced by new bone tissue with constructing osteon structures (Currey, 2002; Fung, 1990). Since the new tissue is constructed under in vivo loadings as the non-deformed state, an indeterminate structure may be generated by the difference of the deformation between the old and new phases. Further, the mechanical properties (e.g. elastic modulus) are also different in these phases (Gibson et al., 2006; Rho et al, 1999). Due to the nonuniform structures in bone tissue, the residual stress may be remained around the replaced region without any external forces.

The aim of this study is to measure the residual stress distribution in the cortical bone of the extremities of vertebrates and to describe the relationships with the osteon structures and mechanical loadings in vivo. In the experiments, the study used the bones of rabbit extremities and measured the residual stresses in the bone axial direction at anterior and posterior positions on the cortical surface; the osteon 
population densities at the sections of the measurement positions were observed by microscopy.

\section{Methods}

Bragg's law, the fundamental equation of X-ray diffraction, is expressed as Eq.

(1).

$2 d \sin \theta=n \lambda$

Using characteristic X-rays with a unique wavelength $\lambda$, Eq. (1) relates the Bragg angle $\theta$ to the interplanar spacing $d$ at a specific lattice plane $(h k l)$.

When bone tissue deforms, the interplanar spacing $d$ of the HAp crystals in the tissue changes. The angle of inclination $\psi$ is defined as the angle between the normal direction of the specimen surface and the diffracted lattice plane. As suggested in Fig. 1, the interplanar spacing in lattice planes under tensile stress $\sigma_{x}$ for the $\psi=90^{\circ}$ direction is larger than that for the $\psi=0^{\circ}$ direction. The relation between $d$ and $\psi$ is affected by the intensity of the stress. 
This study regards cortical bone in the diaphyses of extremities as an orthotropic compound, and Fig. 2 shows the coordinate system employed here on the diaphysis surface. The $x$ and $y$-axes correspond to the bone axial and circumferential directions, with these axes defined as the principal axes. Additionally, it was assumed that the residual stress state in the measurement region was a plane stress, because the used X-rays could only penetrate about $100 \mu \mathrm{m}$ into the specimens and this method only measures the outermost region.

The strain in the HAp lattice plane $\varepsilon^{H}$ is defined as in Eq. (2), where $d_{0}$ is the interplanar spacing and $\theta_{0}(\mathrm{deg})$ is the Bragg angle of the non-strained state.

$$
\varepsilon^{H}=\frac{d-d_{0}}{d_{0}}=-\left(\theta-\theta_{0}\right) \frac{\pi}{180} \cot \theta_{0}
$$

The relation between the bone tissue stress $\sigma$ and the HAp strain $\varepsilon^{H}$ stretched in the bone axial or circumferential direction are described by Eq. (3) (Fujisaki and Tadano, 2007), where $E_{x}^{*}$ and $E_{y}^{*}$ are proportionality coefficients of the ratio of $\sigma$ and $\varepsilon^{H}$ in the respective directions. 


$$
\sigma_{x}=E_{x}^{*} \cdot \varepsilon_{x}^{H}, \sigma_{y}=E_{y}^{*} \cdot \varepsilon_{y}^{H}
$$

The study assumed that the relations between $\sigma$ and $\varepsilon^{H}$ in each direction are described by Eq. (4), where the Poisson's ratio of HAp crystals $v^{*}$ was assumed to be uniform throughout a specimen.

$$
\varepsilon_{x}^{H}=\frac{\sigma_{x}}{E_{x}^{*}}-v^{*} \frac{\sigma_{y}}{E_{y}^{*}}, \quad \varepsilon_{y}^{H}=\frac{\sigma_{y}}{E_{y}^{*}}-v^{*} \frac{\sigma_{x}}{E_{x}^{*}}, \varepsilon_{z}^{H}=-v^{*}\left(\frac{\sigma_{x}}{E_{x}^{*}}+\frac{\sigma_{y}}{E_{y}^{*}}\right)
$$

It is possible to measure the HAp strain $\varepsilon^{H}{ }_{\psi}$ for an angle of inclination $\psi$ as shown in Fig.

2, and $\varepsilon^{H}{ }_{\psi}$ is described with $\varepsilon^{H}{ }_{x}$ and $\varepsilon^{H}$ as in Eq. (5).

$$
\varepsilon_{\psi}^{H}=\varepsilon_{x}^{H} \sin ^{2} \psi+\varepsilon_{z}^{H}\left(1-\sin ^{2} \psi\right)
$$

Using Eq. (4), Eq. (5) can be written as Eq. (6).

$$
\varepsilon_{\psi}^{H}=\frac{1+v^{*}}{E_{x}^{*}} \sigma_{x} \sin ^{2} \psi-v^{*}\left(\frac{\sigma_{x}}{E_{x}^{*}}+\frac{\sigma_{y}}{E_{y}^{*}}\right)
$$


By partial differentiation of Eq. (6) with respect to $\left(\sin ^{2} \psi\right), \sigma_{x}$ can be derived as Eq. (7), and with Eq. (2) $\sigma_{x}$ can be described with the X-ray diffraction angle $2 \theta$ and $\psi$ as in Eq. (8).

$$
\begin{aligned}
& \sigma_{x}=\frac{E_{x}^{*}}{1+v^{*}} \frac{\partial \varepsilon_{\psi}^{H}}{\partial\left(\sin ^{2} \psi\right)} \\
& \sigma_{x}=\left(-\frac{E_{x}^{*}}{2\left(1+v^{*}\right)} \frac{\pi}{180} \cot \theta_{0}\right) \frac{\partial(2 \theta)}{\partial\left(\sin ^{2} \psi\right)} \equiv K_{x} \frac{\partial(2 \theta)}{\partial\left(\sin ^{2} \psi\right)}
\end{aligned}
$$

Finally, the residual stresses in the bone axial direction can be estimated from measurements of a number of diffraction angles at several angles of inclination using Eq. (8). This method is called the $\sin ^{2} \psi$ method of X-ray diffraction (Yamada and Tadano, 2010).

The constant $K_{x}$ in Eq. (8) is called the stress constant, and in this study the stress constant was defined as the product of the tissue elastic modulus $E_{x}$ and the coefficient $k_{x}^{*}$. The $k_{x}^{*}$ value was calculated from Eq. (9), with $\varepsilon_{x}$ the bone tissue strain. 


$$
K_{x}=E_{x} k_{x}^{*}, \quad k_{x}^{*}=\varepsilon_{x} /\left(\frac{\partial(2 \theta)}{\partial\left(\sin ^{2} \psi\right)}\right)
$$

To determine $k_{x}^{*}$, the study used a four-point bending test of a thin bone specimen with X-ray irradiation and $\partial(2 \theta) / \partial\left(\sin ^{2} \psi\right)$ was calculated. Further, $E_{x}$ was measured in a similar four-point bending test.

\section{Experiments}

\subsection{Specimens}

The study used bones of the extremities of adult rabbits (Japanese White Rabbits, female, average age 16.5 weeks, average weight $3.0 \mathrm{~kg}$ ). Figure 3 shows the bones of the extremities: femur, tibia/fibula, humerus, and radius/ulna. Three bone specimens were used in each limb, and the specimens were $60 \mathrm{~mm}$ long in the bone axial direction and cut using a slow speed diamond wheel saw (SBT650: South Bay Technology Co., USA). Although the preparation may release some amount of residual stress from in vivo whole bone, the residual stresses that occurred in these specimens at the osteon level were measured in this study. The bone marrow and the soft tissue around the surfaces were removed and the specimens were kept in saline until just 
before the X-ray measurements.

\subsection{Measurement of residual stress by $X$-ray diffraction}

The measurements were conducted at the anterior and posterior positions at the center of the specimens (Fig. 3). The study defined anterior as the head side and posterior as the tail side of the animal in each limb when the limbs were extended. The anterior and posterior positions in the tibia/fibula correspond to the anterior and posterior positions of the tibia, respectively. Further, in the radius/ulna, the anterior and posterior positions correspond to the anterior position of the radius and the posterior position of the ulna, respectively. The residual stresses were calculated from five measurements at each position.

An X-ray diffractometer (RINT2200: Rigaku Co., Japan) was used to measure $2 \theta$ in Eq. (8) with characteristic Mo-K $\alpha(\lambda \sim 0.071 \mathrm{~nm}) \mathrm{X}$-rays, tube voltage $40 \mathrm{kV}$, and tube current $40 \mathrm{~mA}$. The diffraction profiles were measured between $13.0^{\circ}$ and $17.0^{\circ}$, which includes the diffraction angles of the (211), (112), and (300) lattice planes of HAp crystals. The study defined $2 \theta$ as the angle at the peak position of the profile, and the peak position was determined as the midpoint of the full width at two-thirds maximum intensity of the profile (FWTTM method), as shown in Fig. 4. The $2 \theta$ was 
measured at the following $\psi$ conditions: $0^{\circ}, 10^{\circ}, 20^{\circ}, 30^{\circ}$, and $40^{\circ}$, and $\partial(2 \theta) / \partial\left(\sin ^{2} \psi\right)$ was calculated by the linear least-squares method.

To determine the stress constant $K_{x}$ of the rabbit extremities, the study used a thin specimen $(3 \times 26 \times 0.6 \mathrm{~mm})$ taken from the rabbit femur and a strain gauge (KFG-1N-120-C1-11L3M3R: KYOWA Co., Japan) was bonded to the surface. The strip specimens deformed by the four-point bending (outer span length $=24 \mathrm{~mm}$, inner span length $=12 \mathrm{~mm}$ ) with X-ray irradiation. The conditions of tissue strain $\varepsilon_{x}$ at the surface were $0,500 \mu$, and $1000 \mu$. The value of $\partial(2 \theta) / \partial\left(\sin ^{2} \psi\right)$ was calculated at each strain condition. Then, the $k_{x}^{*}$ value was calculated from the relationship between $\partial(2 \theta) / \partial\left(\sin ^{2} \psi\right)$ and the tissue strain $\varepsilon_{x}$ with Eq. (9). Further, another four-point bending test without the X-ray diffraction system was performed to estimate $E_{x}$ using a material testing machine (Model 4411: INSTRON Co., USA). The $K_{x}$ was calculated from the $k_{x}{ }^{*}$ and $E_{x}$ with Eq. (9).

\subsection{Measurement of osteon population density (OPD)}

The specimens were air dried after the X-ray measurements and then saturated in epoxy resin for 24 hours to be able to easily grind the cross sections. Next, $5 \mathrm{~mm}$ long sections of these specimens were cut out by the diamond wheel saw, with one end 
at the X-ray measurement position. The cross sections were ground by emery papers, and then buffed by a buffing machine (Model 900: South Bay Technology Co., USA). The surface regions of the diaphysis side in the section as shown in Fig. 2 were observed by a microscope (VH5000: KEYENCE Co., Japan). Figure 5 shows a microscopic image of the X-ray measurement position. The observed area was $100 \mu \mathrm{m}$ depths from the outer cortical surface and $700 \mu \mathrm{m}$ widths centering the $z$-axis in the section. The dashed line in Fig. 5 is at about $100 \mu \mathrm{m}$ from the outer surface of the specimen. The black arrows indicate osteons included wholly or partly in the region. In the study, the number of osteons in the region was counted and the osteon population density (OPD) was calculated.

\section{Results}

\subsection{Residual stresses distribution}

Figure 6 shows the distribution of residual stress in the rabbit limb bones. Each bar indicates the average of three specimens in each position, and errors are corresponding standard deviations. Tensile residual stresses were observed at every measurement position. The average stresses at the hindlimb bones and the forelimb 
bones were $210 \mathrm{MPa}$ and $149 \mathrm{MPa}$ respectively, showing that the hindlimb bones were subject to the tensile residual stress 1.4 times greater than that in the forelimb bones. In the femur, the residual stress at the anterior position was larger than at the posterior position, while in the tibia, the stress at the posterior position was larger than at the anterior position. These differences were different in the hindlimb bones. In the forelimb bones, the residual stress at the anterior positions was larger than at the posterior positions.

\subsection{Osteon population density (OPD)}

Figure 7 shows typical microscopic images of the X-ray measurement positions in the hindlimb bones: (a) the anterior position of a femur, (b) the posterior position of a femur, (c) the anterior position of a tibia, and (d) the posterior position of a tibia. In the study, the numbers of osteons were counted and the osteon population densities (OPD) were calculated in the cortical surface region at these sections of the X-ray measurement positions as shown in Fig. 5. It was clear that the OPD was distinct in these images. Table 1 shows the OPD at the X-ray measurement positions. The value of each position was the average of the three specimens. In the femur and humerus, the OPD in the anterior positions were larger than in the posterior positions. In the tibia, the OPD in the 
posterior position was larger than that in the anterior position. Overall, more osteon structures were observed in the positions subjected to higher residual stresses. In the radius/ulna, however, there were no large differences in the OPD here. Figure 8 shows the relationship between the residual stress and the OPD. There was a positive correlation $(r=0.55, P<0.01)$, and the residual stresses correlated with the OPD.

\section{Discussion}

The values of the measured residual stresses depend on the stress constant $K_{x}$, and the stress constant was calculated from the product of the elastic modulus $E_{x}$ and the coefficient $k_{x}^{*}$, as Eq. (9). Here, the $k_{x}^{*}$ stands for the ratio of the tissue strain to the HAp strain as in Eq. (10).

$$
k_{x}^{*}=\frac{1}{\left[-\left(1+v^{*}\right) \frac{360}{\pi} \tan \theta_{0}\right]} \frac{\varepsilon_{x}}{\varepsilon_{x}^{H}} \propto \frac{\varepsilon_{x}}{\varepsilon_{x}^{H}}
$$

Fujisaki and Tadano (2007) showed that the elastic modulus was proportional to $\varepsilon^{H} / \varepsilon$ as in Eq. (11). 


$$
E \propto \frac{\varepsilon^{H}}{\varepsilon} \propto \frac{1}{k^{*}}
$$

Therefore, it can be said that the stress constant $K$ was the same value at the measurement positions and the method used in this study could measure the residual stress distribution without the necessity to consider the distribution of the elastic modulus in the limb bones that we studied.

The study compared the magnitude of the residual stresses to the osteon population density and showed that there were more osteon structures in the positions subject to higher residual stress, as shown in Fig. 8. The differences in the OPD may be explained by the results reported in previous studies (Mason et al., 1995; Skedros et al., 2009), which showed that the OPD and the geometry of osteon structures were correlated with the compression/tension mechanical environments in vivo. Moreover, the differences in the collagen-lamellar organization of these osteons caused these authors to describe them as being distinct osteon "morphotypes", which differ significantly between anterior and posterior cortices in relationship to their habitual strain environments. Mineral crystallite orientation can also differ in these regions, and does not necessarily correlate with collagen fiber orientation or organization (e.g., 
collagen density) (Skedros et al., 2006). In this perspective, the results of the current study suggest that these nonuniform structures of the tissue derived from the osteon formation and the internal organization of these entities down to their nanostructural level might explain the spatial differences in residual stress. Further, this also suggests that the residual stresses being nonuniform may be related to the nonuniformities in the mechanical environments in vivo and the resulting functional adaptation of the bone tissue. The residual stress in the bone is regarded as an epiphenomenon and may be a circumstantial finding of the adapted state.

This study measured the residual stress in the bone axial direction. The measured tensile residual stresses will work to reinforce the bone tissue in compressive loadings in vivo. Adachi et al. (1998) observed residual stresses in vertebral bodies by the cutting method with strain gauges and proposed that the residual stresses might work to allow a stress state to become more uniform. These residual stresses might be important for the mechanical strength of the tissue. For the residual stress distribution in the femurs, the anterior position had larger residual stresses than the posterior position. The previous study of bovine femurs also showed the residual stresses in the anterior position as larger than in the posterior position (Yamada and Tadano, 2010). Here, the femurs of different quadrupeds (e.g. bovine and rabbit femurs) could be subjected to 
similar loadings in vivo, especially the anterior - posterior direction of the bending forces. From the view of the bending forces in vivo and the same variation of residual stress between these species, it would appear that the residual stress variations may be related to the distribution of the mechanical environments in vivo. However, the limb bones used in the current study are subjected to complicate loadings (e.g. compression, bending, and torsion) (Skedros et al., 2009; Vincentelli, 1978). These loadings, especially shear strain, can mess up the attempts to link the residual stress and the tension/compression loadings. Gautier et al. (2000) measured the maximum strain for the bone axial direction in sheep tibia during locomotion using strain gauges; and here the anterior part was subject to tensile and the posterior part to compressive stresses, and further, there were also the effects of the torsion in the strains. The measured residual stresses may not be directly related to the tension/compression strain in vivo. Perhaps the residual stresses may be related to the strain energy density.

The residual stresses would be related to the formation of the osteon structures and/or their collagen/lamellar/crystallite organization. The study showed the correlation between the magnitude of residual stress and the osteon population density, however, it was not very high. Hence, further study is needed to be more clearly the generation process and the biomechanical meanings of these residual stresses. For instance, it will 
be useful to compare the variation of residual stresses with the nonuniform distributions in collagen cross-linking, collagen fiber orientation, osteon morphotypes, and/or CFO heterogeneity. Further, there may be other factors as the formation process arises from osteoblast and osteoclast cell activity. The study here used the bones of rabbit extremities, which were not large, because some experiments on bone structure adaptation due to mechanical stimulation have been conducted using animals of small to medium sizes (Mosley and Lanyon, 1998; Oda et al., 1996; Rubin et al., 2001; Goodship et al., 1979). Comparing the residual stresses of intact and adapted bones would provide more direct data for an elucidation of the relationship between the residual stresses and the bone adaptation.

\section{Acknowledgement}

This work was supported by Grant-in-Aid for Scientific Research (A), MEXT (No. 19200035) and Grant-in-Aid for JSPS Fellows (No. 09J00736).

\section{Conflict of interest statement}

No actual or potential conflicts of interest exist. 


\section{Reference}

Adachi, T., Tanaka, M., Tomita, Y., 1998. Uniform stress state in bone structure with residual stress. Journal of Biomechanical Engineering 120, 342-347.

Almer, J.D., Stock, S.R., 2007. Micromechanical response of mineral and collagen phase in bone. Journal of Structural Biology 157, 365-370.

Currey, J.D., 2002. Bones: structure and mechanics. Princeton University Press, USA, pp. 14-21.

Fujisaki, K., Tadano, S., Sasaki, N., 2006. A method on strain measurement of HAp in cortical bone from diffusive profile on x-ray diffraction. Journal of Biomechanics 39, $579-586$

Fujisaki, K., Tadano, S., 2007. Relationship between bone tissue strain and lattice strain of HAp crystals in bovine cortical bone under tensile loading. Journal of Biomechanics $40,1832-1838$. 
Fung, Y.C., 1990. Biomechanics: Motion, Flow, Stress, and Growth. Springer, Berlin Heidelberg New York, USA, pp. 388-393, 500-503.

Gautier, E., Perren, S.M., Cordey, J., 2000. Strain distribution in plated and unplated sheep tibia an in vivo experiment. International Journal of the Care of the Injured 31, $37-44$.

Gibson, V.A., Stover, S.M., Gibeling, J.C., Hazelwooda S.J., Martin, R.B., 2006. Osteonal effects on elastic modulus and fatigue life in equine bone. Journal of Biomechanics 39, 217-225.

Giri, B., Tadano, S., Fujisaki, K., Sasaki, N., 2009. Deformation of mineral crystals in cortical bone depending on structural anisotropy. Bone 44, 1111-1120.

Goodship, A.E., Lanyon, L.E., McFie, H., 1979. Functional adaptation of bone to increased stress - An experimental study. Journal of Bone and Joint Surgery 61A, $539-546$. 
Gupta, H.S., Seto, J., Wagermaier, W., Zaslansky, P., Boesecke, P., Fratzl, P., 2006.

Cooperative deformation of mineral and collagen in bone at the nanoscale. PNAS 103, $17741-17746$.

Mason, M.W., Skedros, J.G., Bloebaum, R.D., 1995. Evidence of strain-mode-related cortical adaptation in the diaphysis of the horse radius. Bone 17, 229-237.

Mosley, J.R., Lanyon, L.E., 1998. Strain rate as a controlling influence on adaptive modeling in response to dynamic loading of the ulna in growing male rats. Bone 23 , $313-318$.

Oda, J., Sakamoto, J., Aoyama, K., Sueyoshi, Y., Tomita, K., Sawaguchi, T., 1996. Mechanical stresses and bone formation. In: Hayashi, K., Kamiya, A., Ono, K. (Eds.), Biomechanics functional adaption and remodeling. Springer, Tokyo, pp. 123-140.

Rubin, C., Turner, A.S., Bain, S., Mallinckrodt, C., McLeod, K., 2001. Anabolism: Low mechanical signals strengthen long bones. Nature 412, 603-604. 
Rho, J.Y., Zioupos, P., Currey, J.D., Pharr, G.M., 1999. Variations in the individual thick lamellar properties within osteons by nanoindentation. Bone 25, 295-300.

Skedros, J.G., Mendenhall, S.D., Kiser, C.J., Winet, H., 2009. Interpreting cortical bone adaptation and load history by quantifying osteon morphotypes in circularly polarized light images. Bone 44, 392-403.

Skedros, J.G., Sorenson, S.M., Takano, Y., Turner, C.H., 2006. Dissociation of mineral and collagen orientations may differentially adapt compact bone for regional loading environments: results from acoustic velocity measurements in deer calcanei. Bone 39, 143-151.

Tadano, S., Giri, B., Sato, T., Fujisaki, K., Todoh, M., 2008. Estimating nanoscale deformation in bone by x-ray diffraction imaging plate. Journal of Biomechanics 41, 945-952.

Tadano, S., Okoshi, T., 2006. Residual stress in bone structure and tissue of rabbit's 
tibiofibula. Bio-Medical Materials and Engineering 16, 11-21.

Vincentelli, R., 1978. Relation between collagen fiber orientation and age of osteon formation in human tibial compact bone. Acta Anatomica (Basel) 100, 120-128.

Yamada, S., Tadano, S., 2010. Residual stress around the cortical surface in bovine femoral diaphysis. Journal of Biomechanical Engineering 132, 0445031-0445034. 


\section{Figure and Table Legends}

Fig. 1 Interplanar spacing $d$ of HAp crystals at the bone surface in cortical bone tissue under (a) non-strained and (b) tensile loading states. The variation in $d$ with orientation $\psi$ normal to the lattice planes of the HAp crystals is shown in polar coordinates, and the lengths and directions of the vectors in the diagrams show the interplanar spacing and plane-normal direction, respectively. In the non-strained state the interplanar spacing is $d_{0}$. Under tensile loading horizontal to the surface of the specimen, the lattice plane deforms and the interplanar spacing of the lattice planes oriented in the loading direction is the largest and that oriented normal to the surface the smallest.

Fig. 2 Coordinate system on the specimen surface and the X-ray planes for the measurements in the bone axial direction of the diaphysis. The observed area of osteons in the cross-section at the X-ray measurement position is also indicated as a small dark gray area in this figure.

Fig. 3 Specimens of the diaphysis of rabbit extremities, $60 \mathrm{~mm}$ long in the bone axial direction. Measurement positions were anterior and posterior at the center of the diaphyses. The anterior and posterior positions in the tibia/fibula correspond to the anterior and posterior positions of the tibia, respectively. Further, in the radius/ulna, the anterior and posterior positions correspond to the anterior position of the radius and the posterior position of the ulna, respectively.

Fig. 4 Typical X-ray diffraction profile of the cortical bone used in the study and details of the determination of the $2 \theta$ in Eq.(8) as the angle at the peak position of the profile. The diffraction profiles were measured between $13.0^{\circ}$ and $17.0^{\circ}$, which includes the diffraction angles of the (211), (112), and (300) lattice planes of HAp crystals. The influence of the back ground intensity was removed from the profile, and the peak position of the profile was determined as the midpoint of the full width at two-thirds maximum intensity.

Fig. 5 Measurement protocol of the osteon population density (OPD) in the microscopic image at the X-ray measurement position. The observed area was $100 \mu \mathrm{m}$ depths from the outer cortical surface and $700 \mu \mathrm{m}$ widths centering the $z$-axis in the cross-section as shown in Fig. 2. The dashed line is at about $100 \mu \mathrm{m}$ from the outer surface of the specimen. The black arrows indicate osteons included wholly or partly in 
the region. In the study, the number of osteons in the region was counted and the osteon population density (OPD) was calculated. In this image, there were eight osteons in the region and the OPD was 114 counts per a square $\mathrm{mm}$.

Fig. 6 Distribution of the residual stresses in the rabbit limb bones: femur, tibia/fibula, humerus, and radius/ulna. Each bar indicates the average of three specimens in each position, and errors are corresponding standard deviations. The symbol " $A$ " and "P" indicate the anterior and posterior positions in each limb bone, respectively. In the tibia/fibula, the study measured the anterior and posterior positions on the tibia, and no measurements were made on the fibulae. In the radius/ulna, the measurements were made on the anterior position in the radius and the posterior position in the ulna.

Fig. 7 Typical microscopic images at the X-ray measurement positions in the hindlimb bones: (a) anterior in the femur, (b) posterior in the femur, (c) anterior in the tibia, and (d) posterior in the tibia of the same limb. The top of the figures show the outer side of the diaphysis and the central lines in the images indicate $z$-axis. The dashed lines show a depth from the surface of about $100 \mu \mathrm{m}$, and the black arrows indicate osteons included wholly or partly in the region. It was clear that the OPD was distinct in these regions. In the femur, there were more osteons in the anterior position than the posterior positions. In the tibia, there were more osteons in the posterior position than the anterior position.

Fig. 8 Relationship between the residual stress and the OPD in the rabbit limb bones. There was a positive correlation between them $(r=0.55, P<0.01)$.

Table 1 The OPD in the cross section at each measurement position. The observed area of osteons was $100 \mu \mathrm{m}$ depths from the outer cortical surface and $700 \mu \mathrm{m}$ widths as shown in Fig. 5. The value of each position was the average of the three specimens. The unit for the data was the counts per a square $\mathrm{mm}\left(/ \mathrm{mm}^{\wedge} 2\right)$. 
Figures and Tables

Figure 1

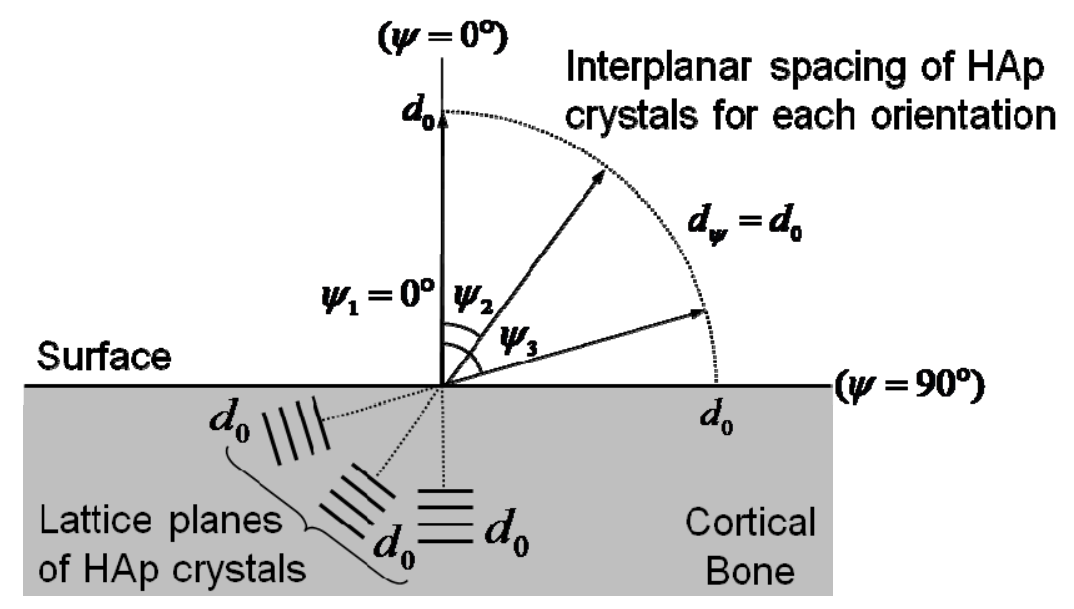

(a) Non-strained state

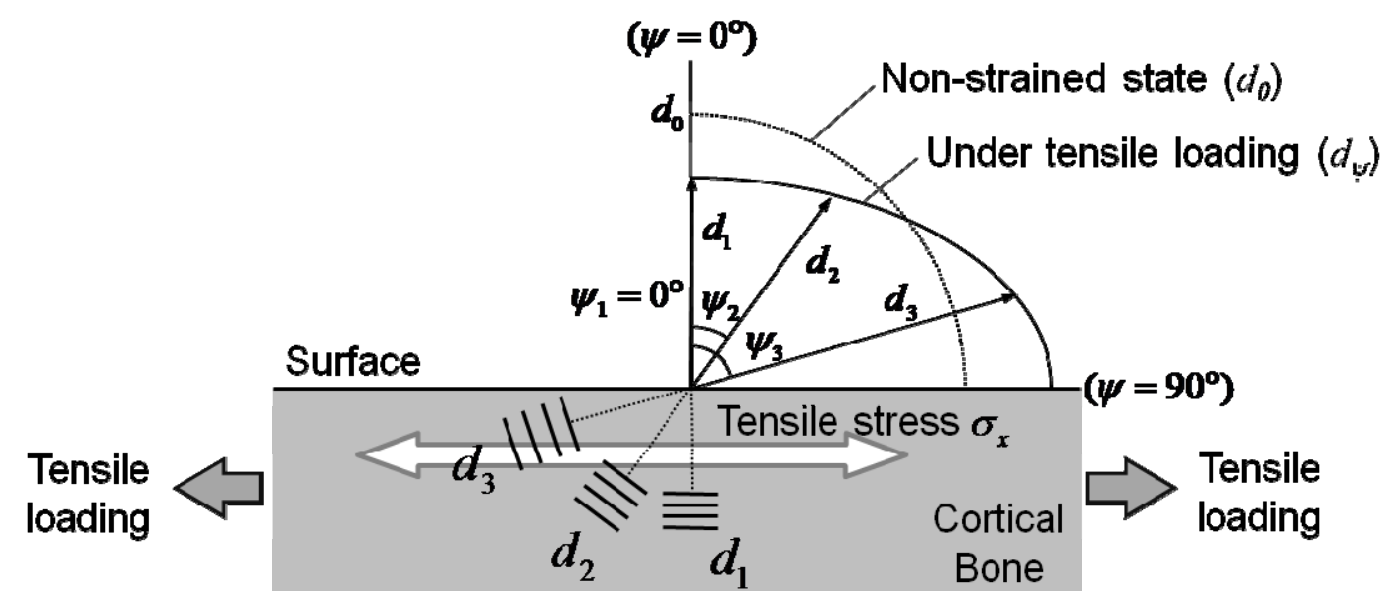

(b) Tensile loading 
Figure 2

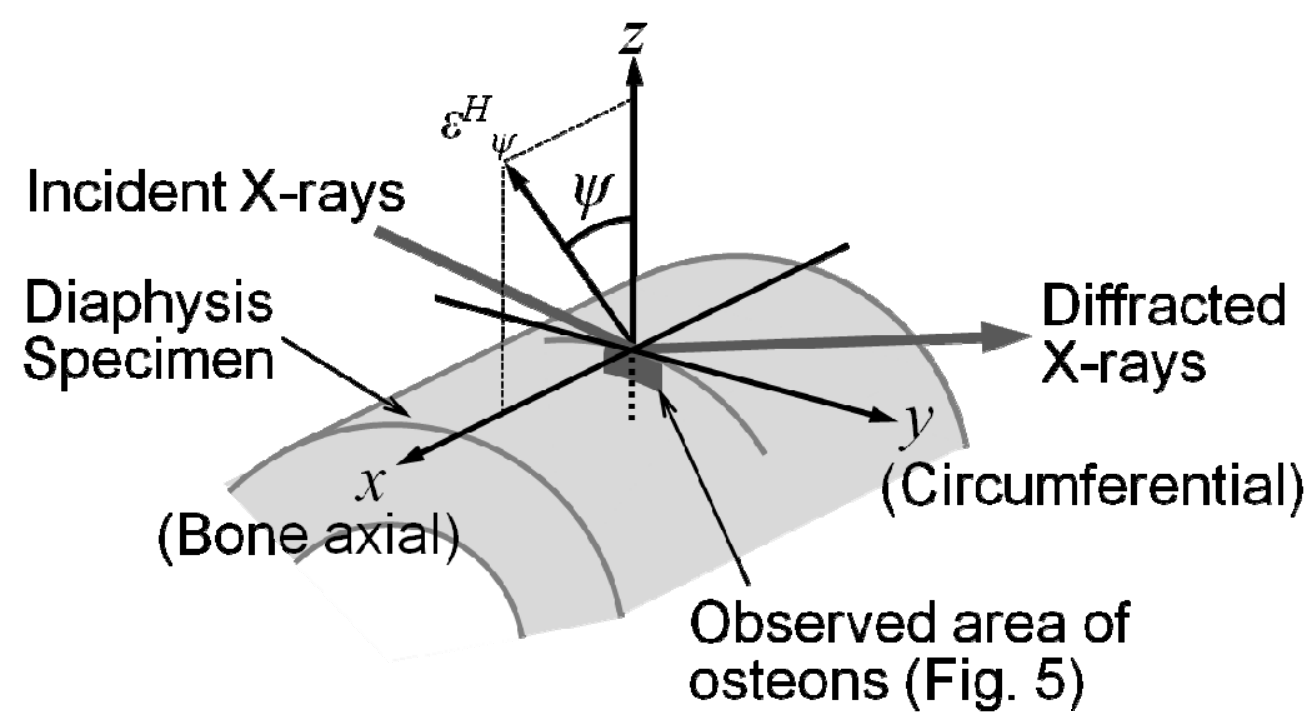

Figure 3

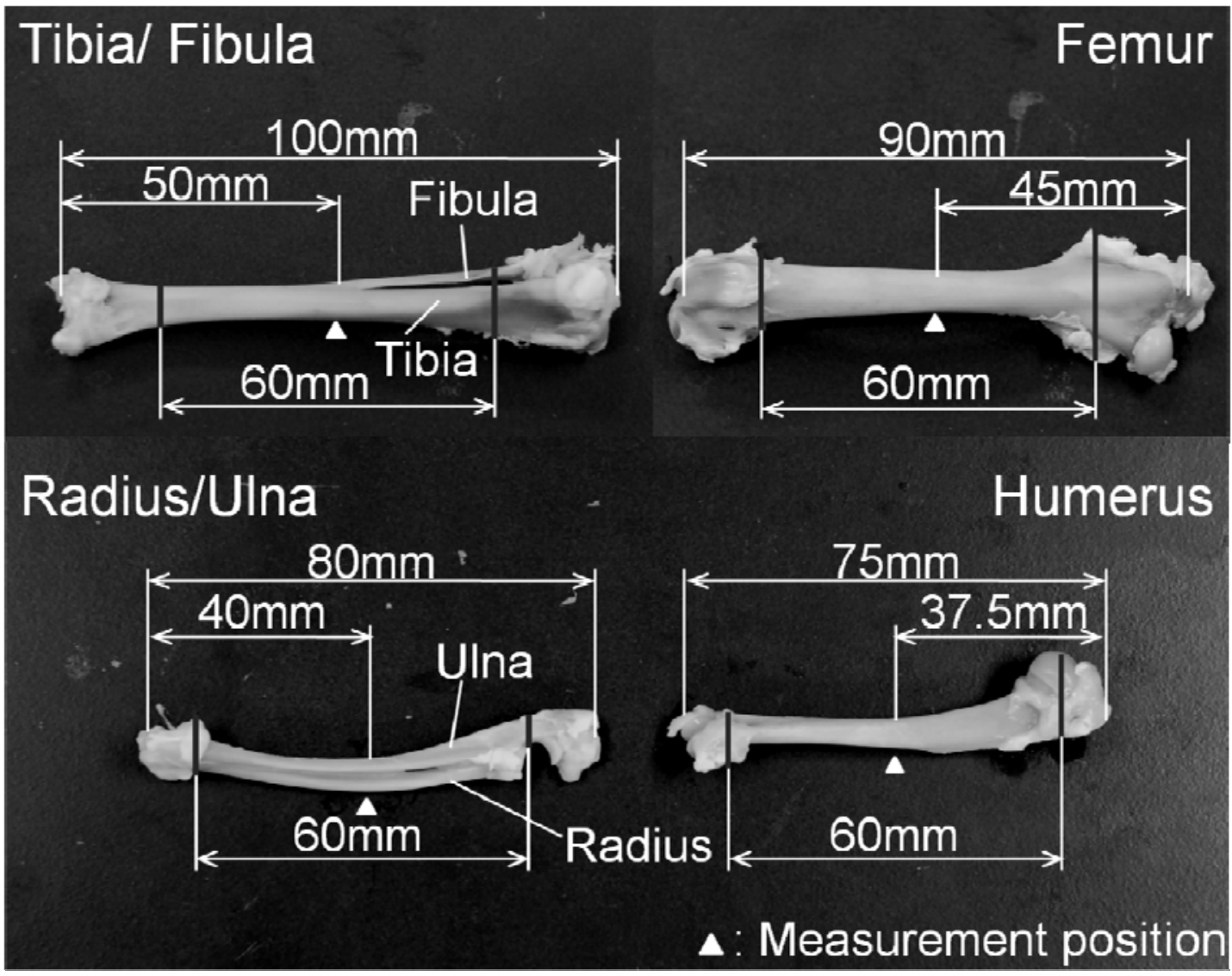


Figure 4

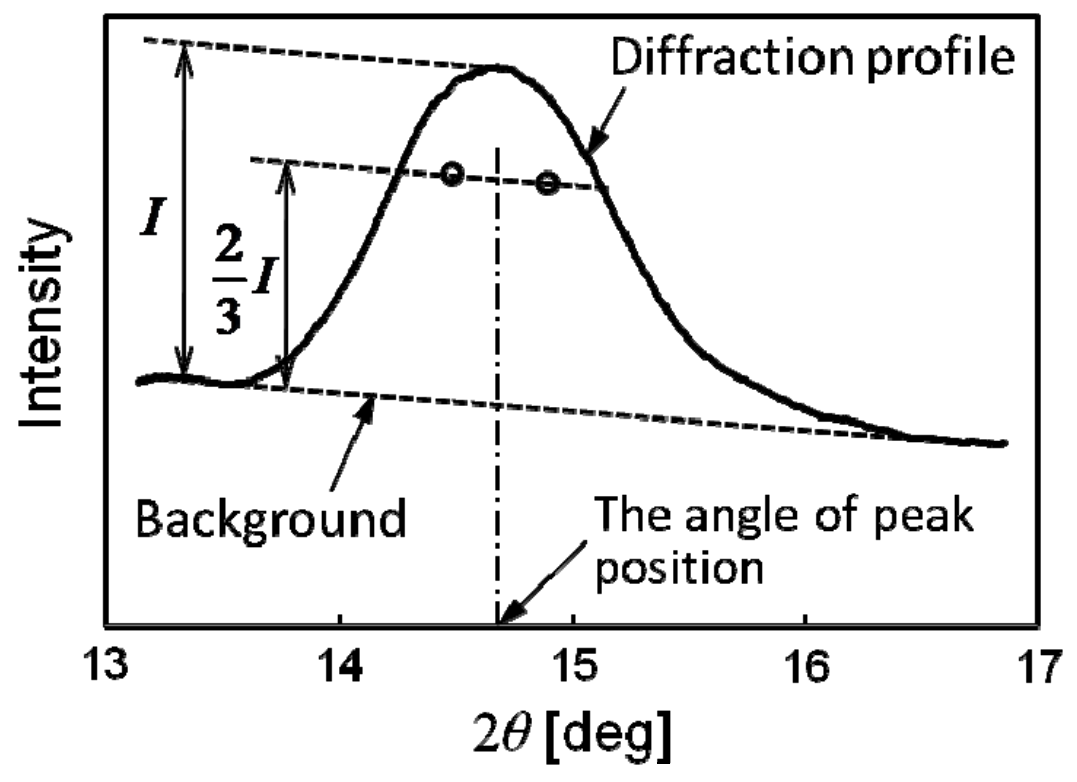

Figure 5

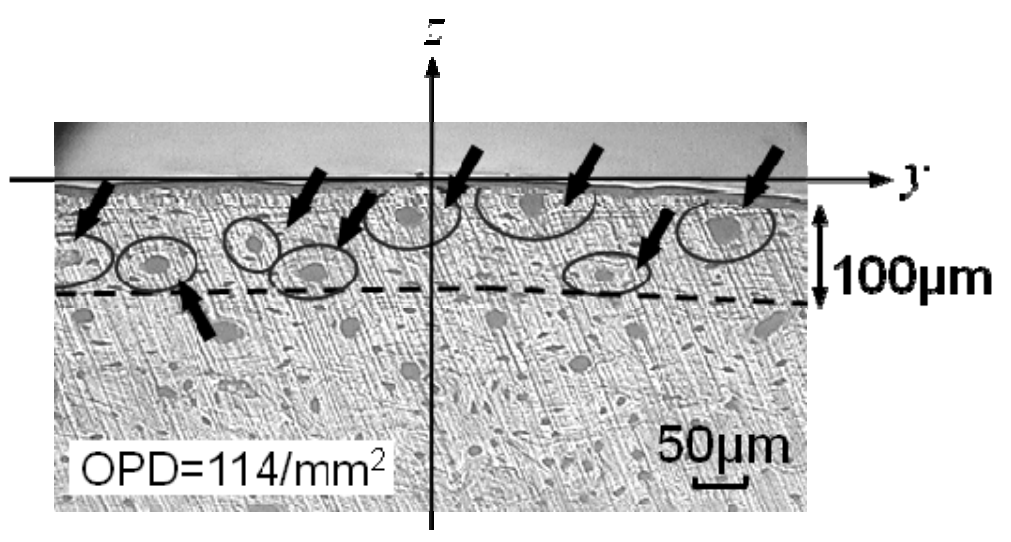


Figure 6

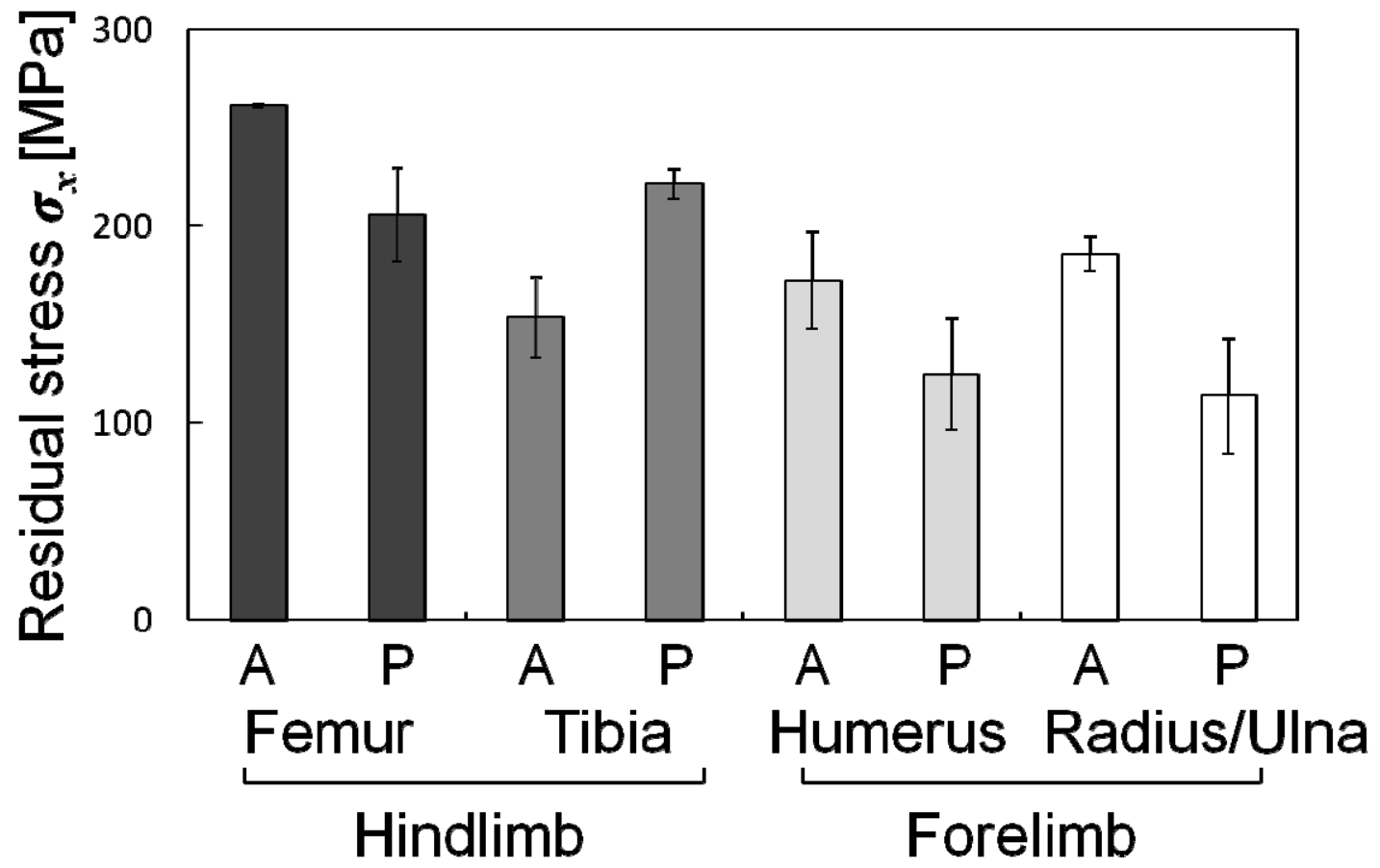


Figure 7

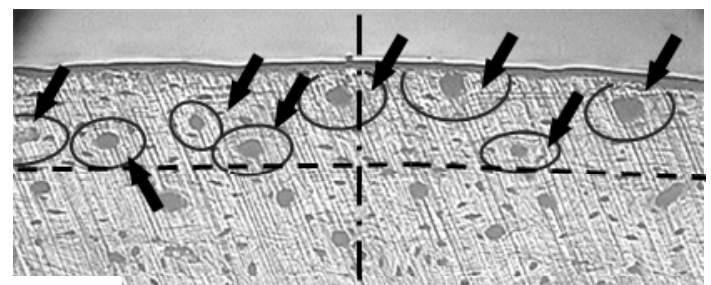

(a)
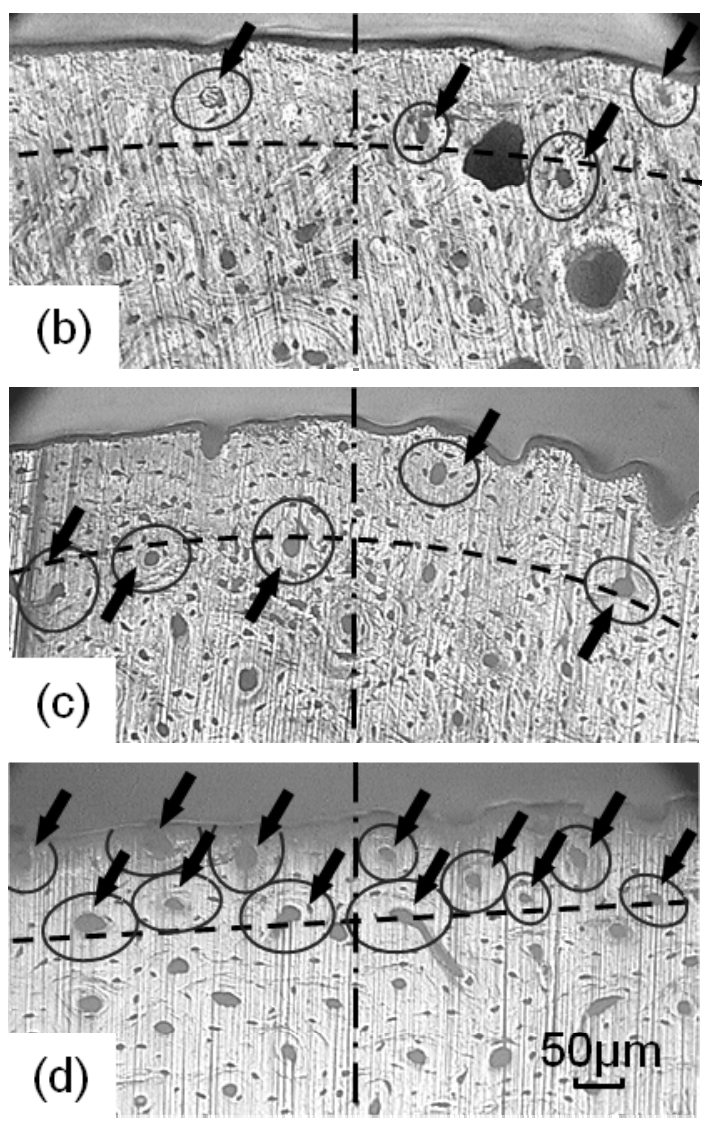
Figure 8

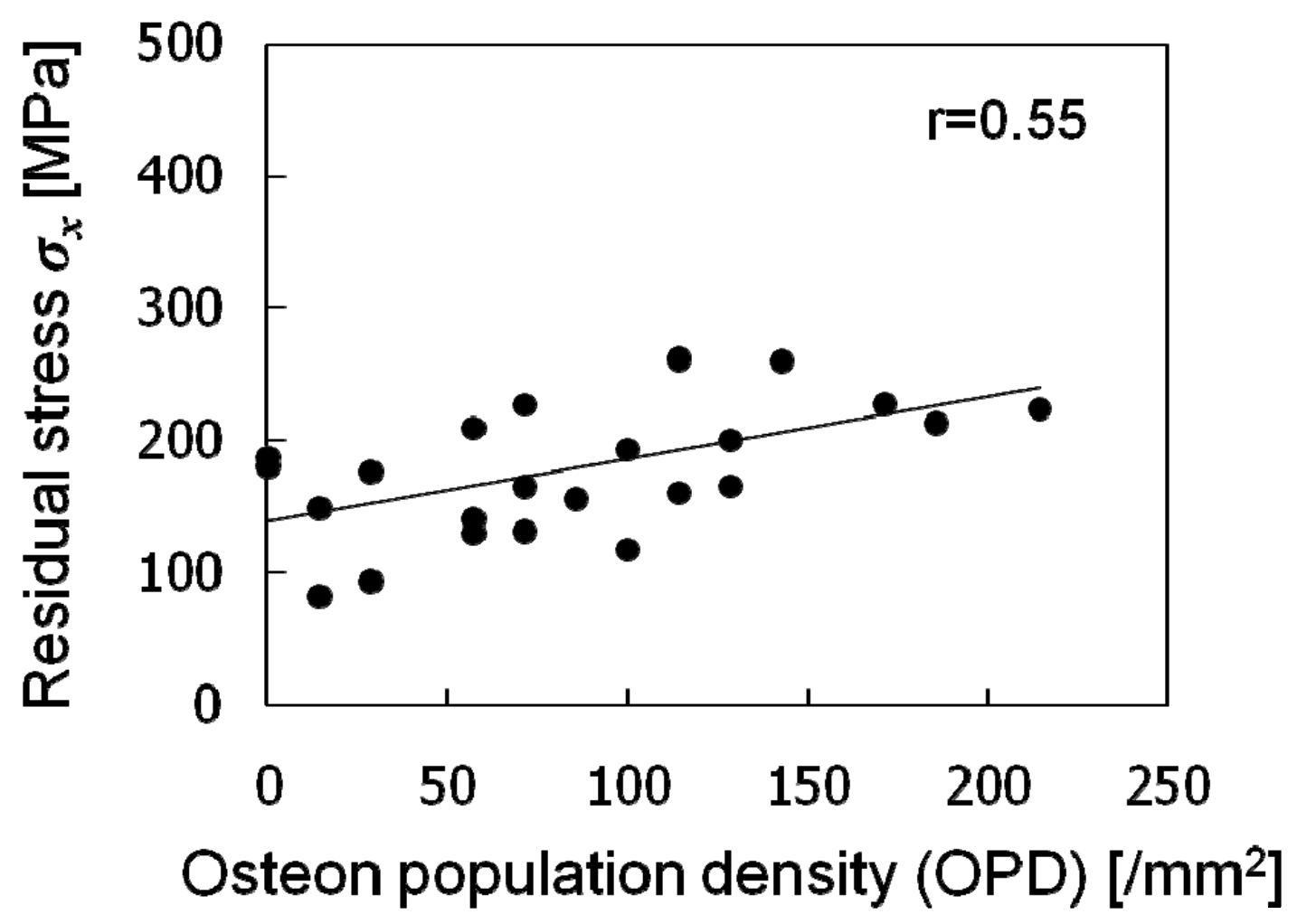

Table 1

\begin{tabular}{c|cccc}
\hline Position & Femur & Tibia & Humerus & Radius / Ulna \\
\hline Anterior & 119 & 94.3 & 119 & 42.9 \\
Posterior & 42.9 & 190 & 47.1 & 61.4 \\
\hline
\end{tabular}

